Tohoku J. exp. Med., 1969, 98, 21-32

\title{
Quantitative Structural Analysis of the Inner Medulla of Rabbit Kidney*
}

\author{
Yasuhiko Sasaki, Tsuneo Takahashi and Norio Suwa \\ The First Department of Pathology (Prof. N. Suwa), \\ Tohoku University School of Medicine, Sendai
}

\begin{abstract}
The inner medulla of rabbit's kidney was examined for the total internal circumferences of Henle's thin segments and collecting tubules at a given medullary height. The results could be approximated by $H(x)=168\left(e^{0.60 x}-1\right)$ for Henle's thin segments and $A(x)=114\left(e^{0,39 x_{-}}-1\right)$ for the collecting tubules. In the expressions $x$ is a distance in mm taken along a medullary ray from the medullary apex to a certain point of the inner medulla, and $H(x)$ and $A(x)$ give the total internal circumferences in mm of Henle's thin segments and collecting tubules respectively on a curved surface defined by $x$. As indicated by different exponents in $H(x)$ and $A(x)$, quantitative predominance of Henle's thin segments over collecting tubules toward the external border of the inner medulla was demonstrated. This was also an expression that in the pile of Henle's loops short loops were overwhelmingly larger in number than long ones.

On the assumption that Henle's loops supply sodium to the interstitial tissue and produce a gradient of sodium concentration rising toward the medullary apex, the structural principle of the inner medulla would counteract the effect. Our histometrical analysis did not consequently favor the counter-current hypothesis. The above equations are to be used in our forthcoming mathematical analysis on the mechanism of urinary concentration.
\end{abstract}

It is generally accepted that the renal medulla of mammals plays an important role in urinary concentration. Schmidt-Nielsen and O'Dell have pointed out that there is a remarkable difference in the ability of urinary concentration among different mammalian species, and the osmolarity of urine is closely correlated with relative medullary thickness. Although their result presents obvious evidence for the participation of the renal medulla in urinary concentration, it is still utterly obscure, how the medullary structure influence the function.

On the other hand, theoretical treatments of medullary physiology have been entirely based on a simplified model with a single Henle's loop, since Hargitay and $\mathrm{Kuhn}^{2}$ proposed the counter-current multiplier hypothesis. However, the renal medulla is constituted by a large number of Henle's loops of extremely different length and ramified collecting tubules. They are piled apparently according to definite structural principle to make the form of the renal medulla as it is. In

Received for publication, January 27, 1969.

* The Mechanism of Urinary Concentration in the Renal Medulla I. 
such a situation, it is obvious that a theoretical conclusion obtained from a single Henle's loop cannot be applied immediately to complete medulla and to final medullary function. The presumed function of individual constituent parts of the renal medulla must be integrated in reference to the structural principle, in order that the validity of a theoretical assumption on renal function is evaluated.

There is to-day a large body of knowledge about the histological and fine structural characters of individual medullary constituents. However, nothing is known about how they are arranged in the space of the renal medulla. For the purpose of understanding the role of the medullary structure in urinary concentration, a quantitative analysis is necessary. The plan of the investigation must be of course controlled by theoretical assumption to be made on the medullary function. In a forthcoming report of ours mathematical derivation is discussed, in which the total internal circumferences of Henle's loops and collecting tubules at a given distance from the papillary apex are used as parameters. Consequently, the present report is concerned with a determination of the above quantities for the purpose of finding some adequate mathematical expressions for them.

It would be most useful, if the quantitative treatment were possible on human kidney, because ample clinical date are available. However, the renal medulla of human kidney is a very complex structure. A number of medullary cones are fused together to share a common papilla. The geometrical configuration of the medulla belonging to a single papilla as determined by reconstruction of serial sections is so complicated and irregular that it discourages every attempt at theoretical treatment. However, the principle of medullary function can be regarded as essentially the same in all mammalin species, and there is no reason to apply different mathematical treatments according to different animal species. Animals with a single renal papilla are feasible to analytical treatment because of the regularity in the medullary shape, and we used rabbits for the present study.

The whole medullary function is shared by outer and inner medullas of different structures. The former has complicated constituents including thick segments of Henle's ascending limb and straight portion of the proximal tubule. The latter, the inner medulla, is much simpler in its structural elements and composed of thin segments of Henle's loops and collecting tubules. An effective analytical study of the medullary function seems to be possible only on the inner medulla. The present study is therefore confined to its structural analysis.

\section{Preliminary Examination}

When a rabbit's kidney is excised, fixed in toto in formalin and macroscopically examined on the largest possible frontal section, a distinct pattern of medullary rays radiating from the papillary apex can be recognized. The sectional surface was photographically magnified and the length of medullary rays from the papillary apex was measured by attaching thin threads along the course of individual medullary rays. The length of the route was practically independent 
of different directions. An example is demonstrated in Fig. 1. In rabbits of about $2,000 \mathrm{~g}$ in weight, the distance along the medullary ray from the papillary apex to the boundary of outer and inner medullas was about $8 \mathrm{~mm}$ and that from the apex to the corticomedullary border was about $11 \mathrm{~mm}$ irrespective of selected routes. In the following part of the present study, the distance taken along the medullary ray from the papillary apex to a certain point is expressed by $x$.

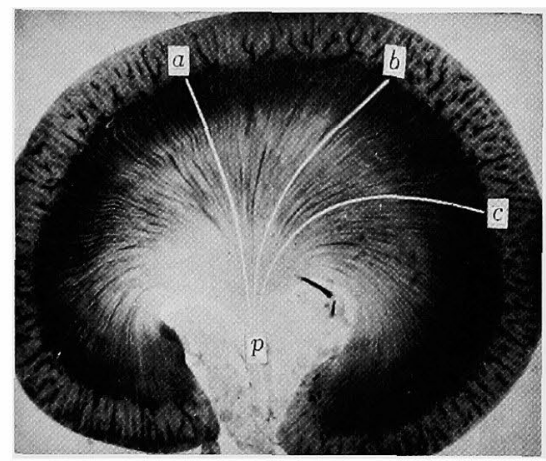

Fig. 1. Medullary rays are demonstrated on the frontal section including the papillary apex of a rabbit's kidney. In this figure, the distance along the medullary ray is taken in three different directions from the papillary apex to the corticomedullary boundary. The measurement gives the following ratio.

$p a: p b: p c=0.86: 0.99: 1.00$.

It is concluded that the distance defined in this way is essentially the same in all the directions.

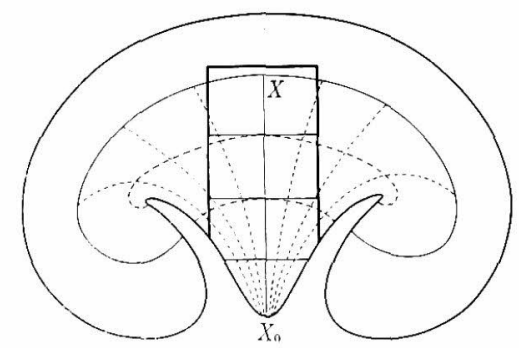

Fig. 2. The orientation of the tissue specimen for serial histological sections is illustrated with thick lines. On the axis $X_{0}-X$ the medullary ray runs straight in its entire course from the medullary apex to the renal cortex. Histological sections were prepared in the direction perpendicular to $X_{0}-X$, so that exact transverse sections of renal tubules were ensured. For some distance from the medullary apex, the cross section of the whole papilla could be demonstrated on histological sections.

Now, the points at the same $x$ on different medullary rays define a curved surface in the medulla, which is henceforth called simply equidistance surface. Under this definition the total medulla can be so imagined that it is made of an infinitely large number of concentrically overlayed equidistance surfaces. It is expected from such a structural principle that the proportion of different constituents to one another is uniform and the function of urinary concentration is the same all over a certain equidistance surface. On this assumption, the 

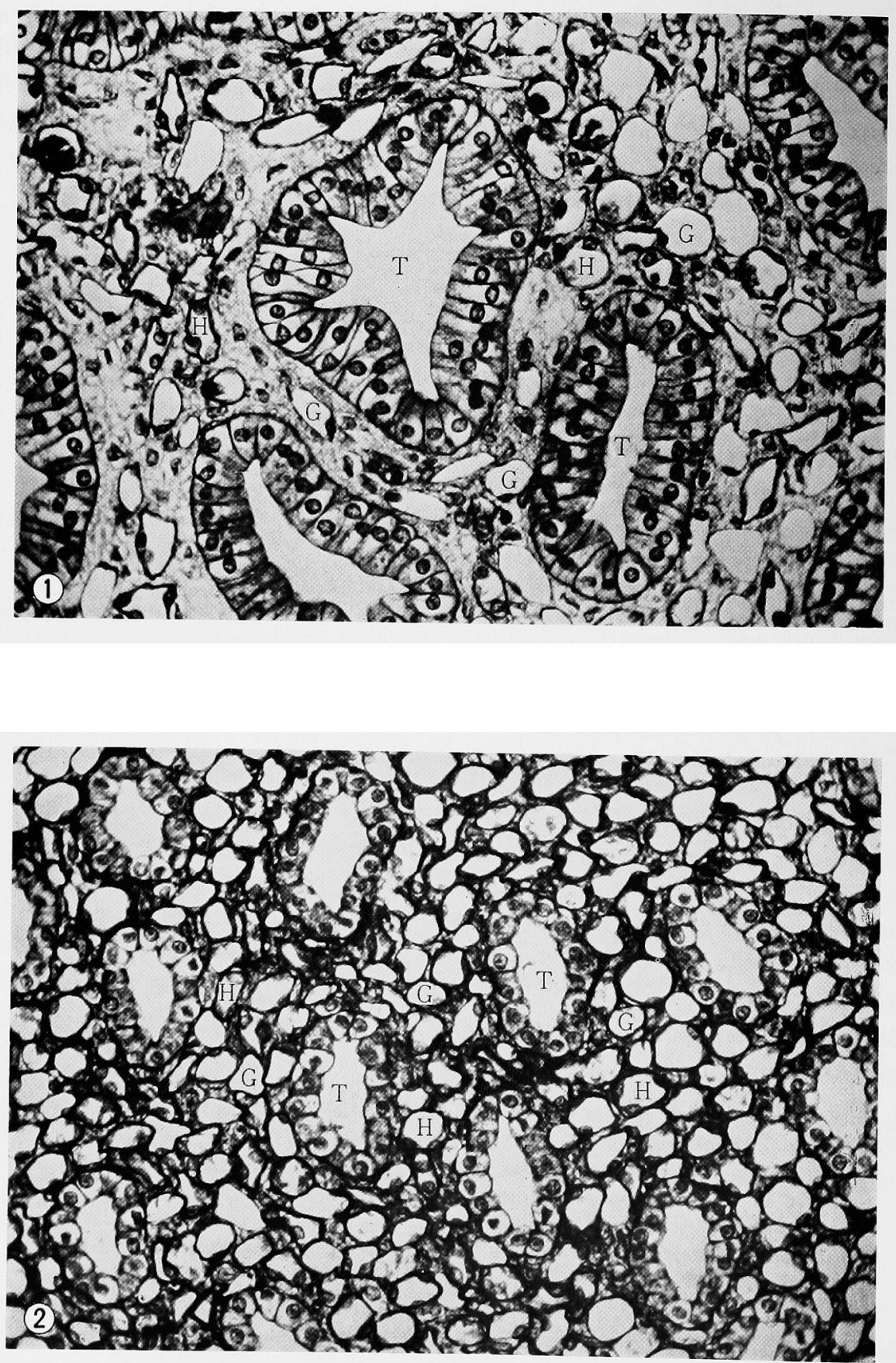


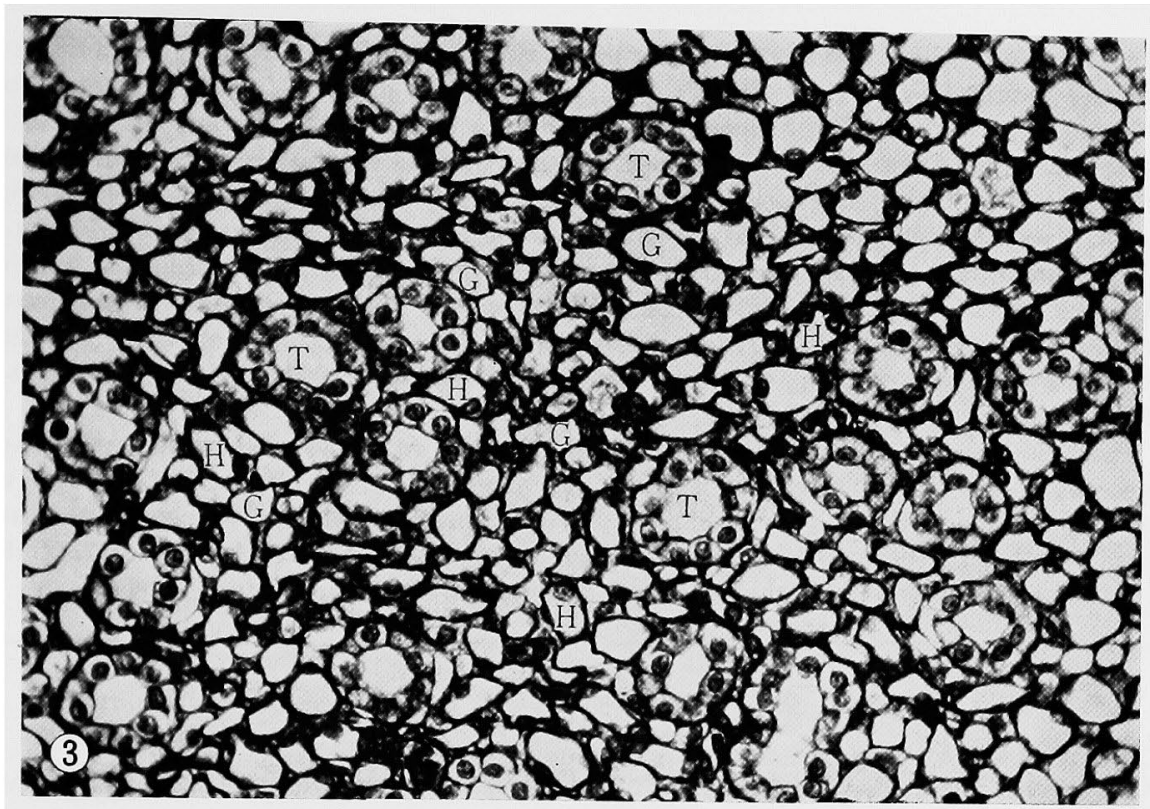

Fig. 3. General histological appearance of the inner medulla of a rabbit's kidney at different heights is presented. From (1) to (3) the sections approach to the corticomedullary border. Only three different constituents are present.

T: Collecting tubule. H: Henle's thin segment. G: Capillary. Note progressive reduction of the size of collecting tubules and capillaries toward the external border of the inner medulla. The size of Henle's loops is essentially the same in all the sections.

information from an arbitrary part of an equidistance surface can be interpreted as representing the whole surface. Consequently, we can take some part out of the medulla, where our histometrical technique is most conveniently applicable.

From Fig. 1 it is clearly recognized that in the middle part of the kidney the medullary ray delineates a straight line in its total course from the medullary apex to the corticomedullary border. Serial histological sections of this part will demonstrate exact transverse tubular sections over the entire course of the tubules in the medulla and will assure easy determination of necessary quantities. Based on this preliminary examination the following method was applied.

\section{Materials and Methods}

From a rabbit weighing about $2,000 \mathrm{~g}$ the left kidney was excised under ether anesthesia. A column of tissue was taken as illustrated in Fig. 2 and immediately fixed in Carnoy's solution. This fixative has an advantage to fix renal tubules and capillaries in a dilated state, which is a requisite for exact histometrical treatment. The tissue specimen was embedded in celloidin-paraffin and serial histological sections of $6 \mu$ in thickness were prepared covering the entire thickness of the inner medulla. The sections were stained with a mixture of Biebrich's scarlet and aniline blue. The former dye stained the cytoplasma of tubular epithelial cells intensively and facilitated the discrimination of Henle's thin segments from capillaries. The latter stain was used for the demonstration of the basement membrane.

General histological pictures are demonstrated in Fig. 3. There was no difficulty in 
identifying the collecting tubules, but the discrimination of Henle's thin segments from capillaries required some training. The major differences of Henle's thin segments from the capillaries were that the former had always a recognizable amount of perinuclear cytoplasma even when general cytoplasmic lining was so attenuated as to be indistinguishable from endothelial cytoplasma, and that epithelial nuclei were larger and clearer than those of capillary endothelial cells. On account of these characteristics discrimination of the two components was possible with good reproducibility after some histological training. Histological discrimination of descending and ascending limbs of Henle's thin segments was utterly impossible. However, the number of loops at a certain medullary height could be immediately obtained from the total number of both limbs, because both limbs must be equal in number.

The relative quantity of each component on an equidistance surface was simply determinable by plane sampling with an adequate eye-piece. In order to obtain the absolute quantity, however, at least one of the different tissue elements must be examined with some other method. In the present study reference was made to the total number and internal circumference of the collecting tubules on an equidistance surface.

\section{Results}

\section{1) Collecting tubule}

Our serial histological sections demonstrated the whole medullary transverse sections from the medullary apex to a height of about $1.35 \mathrm{~mm}$. It was therefore possible in this range to count directly the number of tubular cross sections and to obtain the total number of collecting tubules. Although curved equidistance surfaces did not strictly agree with the planes of the histological sections, the divergence of collecting tubules was only insignificant in this range and the error could be regarded as negligible. Above the height of $1.35 \mathrm{~mm}$, only a part of the inner medulla was given on histological sections, and a direct estimatiom of the total number of collecting tubules was impossible. In this case, the following principle could be applied.

Let the distance along the medullary ray from the medullary apex to a certain point be $x$. We suppose now that the inner medulla was so divided by a sufficiently large number of equidistance surfaces that every two adjacent equidistance surfaces are separated by an equal distance of $\Delta x$. The equidistance surfaces are numbered from the medullary apex to the border of the inner and outer medullas. If the total number of the collecting tubules on the $i$-th equidistance surface is $N_{i}$, that on the $(i+j)$-th equidistance surface $N_{i+j}$ is given by:

$$
N_{i+j}=N_{i}{ }_{i=i}^{i+j-1} \rho_{i}
$$

where $\rho_{i}$ is the ratio of increase in the number of collecting tubules in a distance $\Delta x$ from the $i$-th equidistance surface to the $(i+1)$-th one.

Now, in the range of $1.35 \mathrm{~mm}$ from the medullary apex $N_{i}$ can be directly determined on the $i$-th equidistance surface. On this surface a certain number $m_{i}$ of collecting tubules were randomly taken. By graphical reconstruction from the serial histological sections, the selected number of collecting tubules were pursued for the number $m_{i+1}$ of collecting tubules generated from them after a course of $4 x$. The ratio $m_{i+1} / m_{i}$ gave $\rho_{i}$. On the $(i+1)$-th equidistance surface 
TABLE 1. Determination of the total internal circumference of the collecting tubule at a certain medullary height

(1)

\begin{tabular}{|c|c|c|c|c|c|}
\hline $\begin{array}{c}x \\
(\mathrm{~mm})\end{array}$ & $m$ & $\rho$ & $N_{c}$ & $\begin{array}{c}L_{c} \\
(\mathrm{~mm})\end{array}$ & $\begin{array}{l}L_{c} N_{c} \\
(\mathrm{~mm})\end{array}$ \\
\hline 0.9 & 10 & 4.00 & 110 & 0.2558 & 28.14 \\
\hline 1.8 & $10^{-40}$ & 2.90 & 440 & 0.2078 & 91.45 \\
\hline 2.7 & $29 \times 29$ & 2.34 & 1276 & 0.1688 & 215.42 \\
\hline 3.6 & $50 \div 68$ & 2.06 & 2986 & 0.1371 & 409.48 \\
\hline 4.5 & $47 \quad 103$ & 1.61 & $\begin{array}{ll}6 & 151\end{array}$ & 0.1113 & 685.18 \\
\hline 5.4 & $53 \times 76$ & 1.71 & 9903 & 0.0904 & 896.06 \\
\hline 6.3 & $53-91$ & 2.01 & 16933 & 0.0666 & 1128.71 \\
\hline 7.2 & $51^{\vee} 107$ & 1.53 & 34036 & 0.0597 & 2032.22 \\
\hline 8.1 & $* 78$ & & 52075 & 0.0485 & 2525.68 \\
\hline
\end{tabular}

(2)

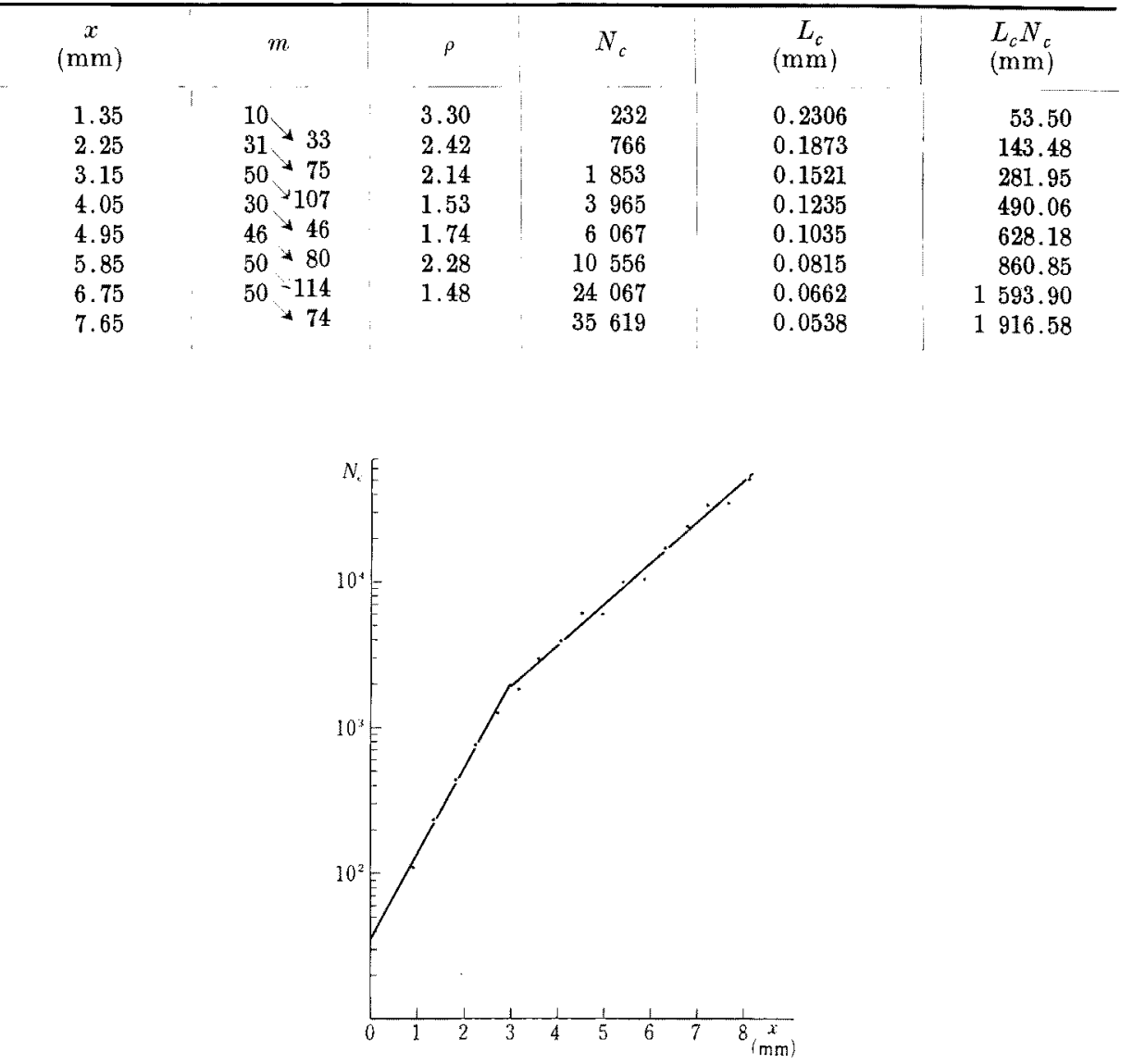

Fig. 4. The number $N_{\text {c }}$ of the collecting tubule as a function of $x$ is demonstrated on a milogarithmic coordinate system. Linear regression is observed. Note a deflection of the regression line at $x=3 \mathrm{~mm}$. 
again a certain number of collecting tubules were taken and the same procedure was repeated to obtain $\rho_{1+i}$. In this way, the total number of collecting tubules could be estimated on all the equidistance surfaces. The distance $x$ of an $(i+j)$-th equidistance surface from the medullary apex is given by $x=(i+j) \Delta x$. Consequently, the total number $N_{c}$ of collecting tubules could be defined by a function of $x$.

The results of determination is demonstrated in Fig. 4. On a semilogarithmic coordinate system a distinct linear regression was observed. This indicates that the total number $N_{c}$ of collecting tubules is defined by an exponential function of $x$. At the same time, a remarkable deflection of the regression line was found at $x=3 \mathrm{~mm}$. Accordingly, $N_{c}$ must be approximated by two exponential functions with different exponents according to whether $x$ is larger or smaller than $3 \mathrm{~mm}$.

As will be seen in a forthcoming report of ours, the quantity required of the collecting tubule in our theoretical treatment is not the total number of collecting tubules but the total internal circumference of collecting tubules as a function of $x$. For this purpose the internal circumference of individual collecting tubules must be known. Exact transverse sections of collecting tubules at different $x$ were depicted under an adequate magnification and the internal circumference of each tubular section was measured by attaching a thin thread. The result of measurements is presented in Fig. 5. A distinct linear regression is demonstrated on a semilogarithmic coordinate system, and the internal circumference $L_{c}$ in $\mathrm{mm}$ of a collecting tubule is given by an exponential function of $x$ as:

$$
L_{c}=0.315 e^{-0.231 x} .
$$

From a product $N_{c} L_{c}$ the total internal circumference $A(x)$ at a certain $x$ can be obtained. In the present study, the results of measurements as given in Table 1 were used as the estimates of $N_{c} . \quad L_{c}$ was obtained from the above regression equation. The values of $A(x)$ determined in this way are plotted on a semilogarith-

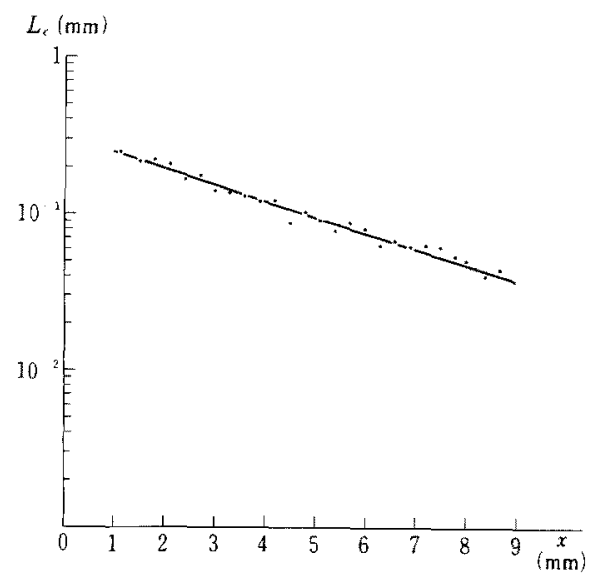

Fig. 5. Internal circumference $L_{c}$ of individual collecting tubules is presented against $x$ on a semilogarithmic coordinate system. Note distinet linear regression. Wach dot represents the mean of measurements on about 10 transverse sections of collecting tubules from the same histological section. 


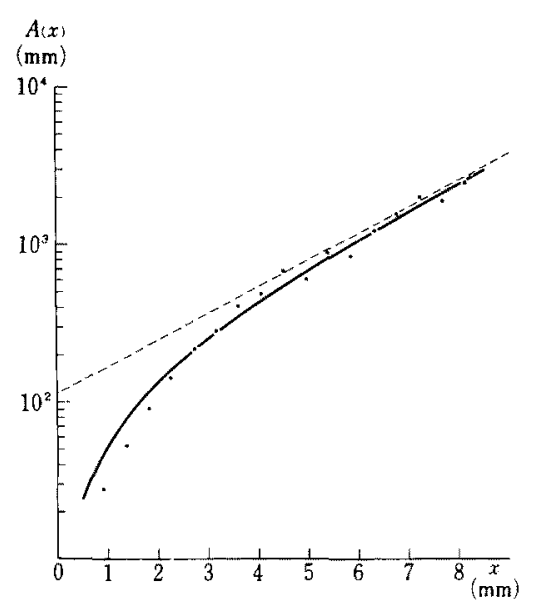

Fig. 6. The total internal circumference of the collecting tubules is approximated by a function of $x$ in the form of $A(x)=A\left(e^{n x}-1\right)$ on a semilogarithmic coordinate system. The dotted line represents $A \epsilon^{a x}$.

mic coordinate system in Fig. 6. The deflection of the regression line at $x=3 \mathrm{~mm}$ in Fig. 5 is naturally reproduced in Fig. 6, and $A(x)$ is to be expressed by two different exponential functions of $x$. However, it is rather convenient for a theoretical treatment to approximate the whole range with a single function of $x$. Further it is required that $A(x)$ becomes zero at $x=0$, because every medullary constituent has to disappear at the medullary apex. These requirements are practically satisfied, when a function of the following form is used:

$$
A(x)=A\left(e^{a x}-1\right),
$$

where $A$ and $a$ are constants. The values of $A$ and $a$ were estimated from the data of Table 1 and the following equation was determined:

$$
A(x)=114\left(e^{0.39 x}-1\right)
$$

the unit being mm. The curve of the equation is given in Fig. 6. Because the equation has a positive exponent, $A(x)$ increases with increasing $x$. This corresponds to progressive branching of the collecting tubules toward the renal cortex. Although individual tubules become smaller, the reduction in size is overcome by increase in number.

\section{2) Henle's loop}

With the determination of $A(x)$ the estimation of the total circumference $H(x)$ of Henle's thin segments is essentially facilitated. This would be most simply to perform by means of line sampling. An eye-piece with a straight line is superposed on the microscopical field. The ratio of the intersections of the sampling line with the internal border of each tubular system gives the ratio of the total circumference of the two systems after a sufficiently large number of trials. In this procedure, however, the total number of Henle's thin segment is not explicitly revealed. Because the quantity is also to be used in the forthcoming theoretical treatment, we estimated the total number $N_{k}$ of Henle's thin segments and the internal 
circumference $L_{h}$ of individual segments separately. The latter quantity was determined by the same method as in the case of $L_{c}$. It was found that $L_{k}$ was a constant independent of $x$ and could be expressed as:

$$
L_{k}=0.0548 \pm 0.0020 \mathrm{~mm}
$$

at $5 \%$ level. The estimation of $N_{h}$ was easy, when an eye-piece with lattice was superposed on the microscopical field and the number of each of Henle's thin segments and collecting tubules was counted in a certain surface area. The result gave a ratio of $N_{h}$ to $N_{c}$, and $N_{h}$ was immediately obtained when $N_{c}$ was known, and from the product $L_{h} N_{h}$ the total internal circumference of Henle's thin segments at a certain $x$ could be determined. The result is shown in Fig. 7 . The general pattern of the figure is similar to that of Fig. 6, and linear regression

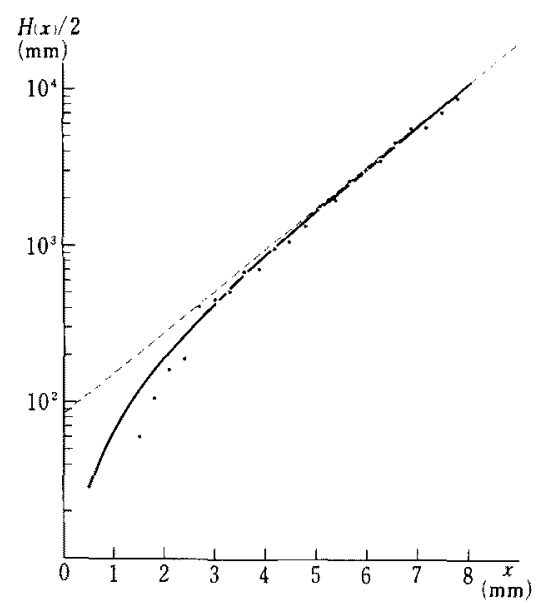

Fig. 7. A half of the total internal circumference of Henle's thin segments is demonstrated on a semilogarithmic coordinate system and approximated by $H(x) / 2=H\left(e^{h x}-1\right) / 2$. The ordinate corresponds accordingly to the total internal circumference of either descending or ascending limb at $x$. The dotted line represents $H e^{h x} / 2$.

with a deflection of the regression line at $x=3 \mathrm{~mm}$ is clearly demonstrated. The approximation of the result is accordingly possible with:

$$
H(x)=H\left(e^{h x}-1\right),
$$

$H$ and $h$ being constants. From the measurements $H$ and $h$ could be calculated and the following equation was obtained:

the unit being $\mathrm{mm}$.

$$
H(x)=168\left(e^{0.60 x}-1\right),
$$

$H(x)$ increases rapidly with increasing $x$. Since $L_{h}$ is a constant, this increase is brought about by an increasing number of short loops. The pile of Henle's loops is characterized by predominance of short loops.

The most important difference of $H(x)$ from $A(x)$ was a distinctly higher exponent in the former than that in the latter. The value of $h$ was 0.60 in contrast 


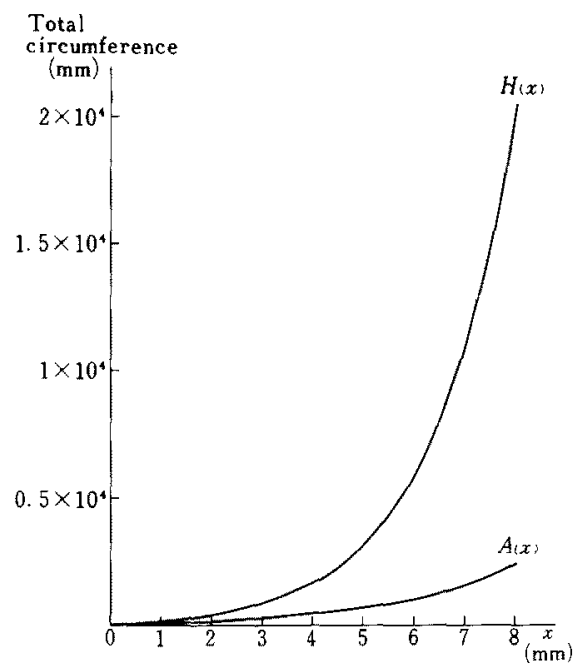

Fig. 8. Total internal circumference of Henle's thin segments $H(x)$ is compared with that of the collecting tubules $A(x)$ on a cartesian coordinate system. Note a rapid increase of $H(x)$ with increasing $x$.

to 0.39 of $a$. The significance of this difference is convincingly revealed on a cartesian coordinate system. In Fig. 8 steep rise of $H(x)$ with increasing $x$ is really remarkable. At $x=3 \mathrm{~mm}$ the ratio $H(x) / A(x)$ is about 3.3 , while it is elevated to about 8 at $x=8 \mathrm{~mm}$. The quantitative predominance of Henle's loops over collecting tubules toward the external border of the inner medulla is the most important result of the present investigation.

\section{Comments}

With the determination of the equations for Henle's thin segments and collecting tubules the aim of the present study is essentially achiever. The meaning of the result can be sufficiently evaluated only after the derivation of a mathematical model on medullary function, which is to be discussed in a forthcoming report of ours. In this connection, only a brief intuitive comment seems appropriate. Since the report of Wirz, ${ }^{3}$ it has been confirmed by a number of investigators that sodium concentration is remarkably elevated toward the medullary apex not only in the medullary interstitial tissue but also in Henle's loops. In the formation of such a gradient of sodium concentration the major role has generally been attributed to Henle's loops as a counter-current multiplier system. According to this hypothesis, Henle's loops act as a system supplying sodium to the interstitial tissue.

The final sodium concentration of the interstitial tissue is the result of interaction of sodium and water transport from both Henle's loops and collecting tubules. The total sodium quantity afforded depends not only on the transport activity of individual tubules but also on the number of tubules. In reference to sodium 
transport the structural principle of Henle's loops as revealed in the present study would not immediately support the part of Henle's loops as a sodium supplying system: the quantitative predominance of the loops over the collecting tubules toward the external border of the inner medulla would counteract the effect to produce a gradient of sodium concentration rising toward the medullary apex.

Our histometrical analysis of the renal medulla made it rather probable that Henle's loops would deprive the interstitial tissue of sodium. On this assumption, the pile of Henle's loops would be effective in producing the pattern of experimentally observed gradient of sodium concentration. The view is to be substantiated by our forthcoming theoretical treatment, in which the interaction of Henle's loops and collecting tubules is discussed analytically.

The present study is based entirely on the exeised kidney, where blood and urinary flow has completely ceased. It is probable that in the living organism renal tubules have larger lumina than those observed in our histological sections on account of intratubular pressure. Further it is to be noticed that the shrinkage of tissue in the course of paraffin embedding was not taken into consideration in the present study. We must have estimated $H(x)$ and $A(x)$ smaller than they are in the living organism. However, in our model of medullary function the ratio $H(x) / A(x)$ will be of significance, but not the absolute values of $H(x)$ and $A(x)$. Possible errors of the above-mentioned sources would be compensated to the major part in the ratio, and we did not apply any correction to our histometrical results to reproduce the state in the living animal.

\section{References}

1) Schmidt-Nielsen, B. \& O' Dell, R. Structure and concentrating mechanism in the mammalian kidney. Amer. J. Physiol., 1961, 200, 1119-1124.

2) Hargitay, B. \& Kuhn, W. Das Multiplikationsprinzip als Grundlage der Harnkonzentrierung in der Niere. Z. Elehtrochem., 1951, 55, 539-558.

3) Wirz, H. Der osmotische Druck des Blutes in der Nierenpapille. Helv. physiol. Acta, $1953,11,20-29$. 